\title{
HALL-CLOSURE AND PRODUCTS OF FITTING CLASSES
}

\author{
OWEN J. BRISON \\ (Received 29 July 1980; revised 2 February 1981) \\ Communicated by D. E. Taylor
}

\begin{abstract}
The Fitting class (of finite, soluble, groups), $\mathfrak{F}$, is said to be Hall $\pi$-closed (where $\pi$ is a set of primes) if whenever $G$ is a group in $\mathfrak{F}$ and $H$ is a Hall $\pi$-subgroup of $G$, then $H$ belongs to $\mathfrak{F}$. In this paper, we study the Hall $\pi$-closure of products of Fitting classes. Our main result is a characterisation of the Hall $\pi$-closed Fitting classes of the form $\mathfrak{F} \# \mathscr{S}_{\text {. }}$ (where $\mathcal{S}_{\text {. denotes the so-called smallest normal }}$ Fitting class), subject to a restriction connecting $\pi$ with the characteristic of $\mathfrak{F}$. We also characterise those Fitting classes $\mathfrak{F}$ (respectively, $\mathfrak{D}$ ) such that $\mathfrak{X} \# \mathfrak{F}$ (respectively, $\mathfrak{D} \# \mathfrak{X}$ ) is Hall $\pi$-closed for all Fitting classes $\mathfrak{X}$. In each case, part of the proof uses a concrete group construction. As a bonus, one of these constructions also yields a "cancellation result" for certain products of Fitting classes.
\end{abstract}

1980 Mathematics subject classification (Amer. Math. Soc.): 20 D 10, 20 D 25, 20 E 22.

\section{Introduction}

All groups considered in this paper are finite and soluble, and all classes of groups considered are subclasses of the class $\mathbb{S}$ of all finite soluble groups. A Fitting class $\mathfrak{F}$ is a non-empty class of groups such that (1) if $G \in \mathfrak{F}$ and $N \unlhd G$, then $N \in \mathfrak{F}$, and (2) if $G=N_{1} N_{2}$ where $N_{i} \unlhd G$ and $N_{i} \in \mathfrak{F}$, then $G \in \mathfrak{F}$.

Let $\pi$ be a set of prime numbers. The Fitting class $\mathscr{F}$ is said to be Hall $\pi$-closed if whenever $G \in \mathfrak{F}$ and $H$ is a Hall $\pi$-subgroup of $G$, then $H \in \mathfrak{F}$, while $\mathfrak{F}$ is said to be Hall-closed if it is Hall $\tau$-closed for all sets $\tau$ of primes. Bryce and Cossey (1975) showed that the so-called smallest normal Fitting class, denoted by $\widetilde{S}_{*}$, is Hall-closed, and this result serves as a starting point for some of our work here. More recently, Cusack (1980) has given a criterion for the Hall

C Copyright Australian Mathematical Society 1982 
$\pi$-closure of Fitting classes in terms of the "join" operation (see Section 8 below), although we will not be concerned with this aspect here.

If $\pi$ is a set of primes, then $\pi^{\prime}$ denotes the complementary set of primes and $\mathfrak{S}_{\pi}$ denotes the class of all (finite, soluble) $\pi$-groups. The Fitting class $\mathfrak{F}$ is clearly Hall $\pi$-closed if either $\mathfrak{S}_{\pi} \subseteq \mathfrak{F}$ or $\mathfrak{F} \subseteq \Im_{\pi^{\prime}}$. These two conditions are both cases of the single condition

$$
\mathfrak{F} \cap \mathfrak{S}_{\pi}=\mathfrak{S}_{\pi \cap a}, \quad \text { where } \sigma=\operatorname{char}(\mathfrak{F}) \quad \text { (see below), }
$$

and it is not hard to check that $\mathfrak{F}$ is Hall $\pi$-closed if it satisfies $(\gamma)$. Although there exist many Hall $\pi$-closed classes which do not satisfy $(\gamma)$, for example the class $\mathfrak{S}_{*}$, and the class $\Re$ of nilpotent groups, the results of this paper indicate that classes satisfying $(\gamma)$ play an important part in the Hall $\pi$-closure of certain products of Fitting classes. If $\pi$ is the set of all primes, or if $\pi$ contains only one prime, then any Fitting class is Hall $\pi$-closed, and so we often exclude these cases.

The Fitting class product $\mathfrak{F} \#(\mathbb{S}$ of Fitting classes $\mathfrak{F}$ and $\mathbb{B}$ (see Section 2) has occasioned considerable interest, especially in the case $\mathbb{S}=\mathfrak{S}_{*}$. The fundamental properties of this product were developed by Cossey (1975), and further results have been proved by Beidleman (1977), Hauck (1979a) and Hauck (1979b). Here, we prove a number of results concerning the Hall $\pi$-closure of products of Fitting classes, taking classes of the form $\mathfrak{F} \# \mathbb{S}_{*}$ as our chief interest.

Our main result, Theorem A, carries on the investigation of products of the form $\mathfrak{F} \# \mathfrak{S}_{*}$ by determining which of these classes are Hall $\pi$-closed, subject to a restriction connecting $\pi$ with the characteristic of $\mathfrak{F}$. Examples are given to show that some restriction is necessary for the result to hold.

Theorems B and D are concerned with the Hall $\pi$-closure of general products. Theorem B determines those Fitting classes $\mathfrak{X}$ such that $\mathfrak{X} \mathbb{F}$ is Hall $\pi$-closed for all Fitting classes $\mathfrak{F}$, while Theorem $D$ determines those Fitting classes $\mathfrak{X}$ such that $\mathfrak{F} \# \mathfrak{X}$ is Hall $\pi$-closed for all Fitting classes $\mathfrak{F}$.

In each of Theorems A, B and D, one direction of the proof is relatively easy, if not trivial, while the other direction uses a concrete group construction. As something of a bonus, the construction used for Theorem B yields several further results. One of these results, Theorem $\mathrm{C}$, is a cancellation property for certain products of Fitting classes, which generalizes a theorem of Hauck (1979a).

The paper is divided into eight sections. The second contains background material for the convenience of the reader, while the third gives three lemmas which we shall need for Theorem A. The fourth section outlines several methods for constructing Hall $\pi$-closed products. Section five is devoted to the proof of Theorem A, while Section six contains the proofs of Theorems B and C. 
Theorem D is Section seven, while the final section mentions Cusack's work, for completeness, and discusses the general case of Theorem A.

Certain results in this paper are developments of results in the author's Ph.D. thesis at The University of Warwick. We would like to thank Dr. Trevor Hawkes for his supervision of that thesis, and The Royal Commission for the Exhibition of 1851 for financial support. We would also like to thank the referee for shortening considerably our original Construction 5.3.

\section{Background material}

Let $\mathfrak{F}$ be a Fitting class and $G$ be a group. The $\mathfrak{F}$-radical of $G$, denoted by $G_{\mathfrak{F}}$, is the largest normal subgroup of $G$ which lies in $\mathfrak{F}$. The characteristic of $\mathfrak{F}$, denoted by $\operatorname{char}(\mathfrak{F})$, is the unique set $\pi$ of primes such that $\mathfrak{N}_{\pi} \subseteq \mathfrak{F} \subseteq \mathfrak{S}_{\pi}$, where $\mathfrak{R}_{\pi}=\mathfrak{R} \cap \mathfrak{S}_{\pi}$ : that such a set exists is shown in Hartley (1969). We say that $\mathfrak{F}$ is closed under taking subdirect products if whenever $N_{1}, N_{2} \unlhd G$ with $G / N_{1}, G / N_{2} \in \mathfrak{F}$, then $G /\left(N_{1} \cap N_{2}\right) \in \mathfrak{F}$. The set of all prime numbers is denoted by $\mathbf{P}$, the set of natural numbers by $\mathbf{N}$. If $p \in \mathbf{P}$ and $\pi \subseteq \mathbf{P}$ then $\operatorname{Syl}_{p}(G)$ and $\mathrm{Hall}_{\pi}(G)$ denote the set of Sylow $p$-subgroups and the set of Hall $\pi$-subgroups, respectively, of $G$; further, $O_{p}(G)$ will denote the largest normal $p$-subgroup of $G$. If $n \in \mathrm{N}$, then $\operatorname{char}(n)$ will denote the set of prime divisors of $n$, while $C_{n}$ will denote a cyclic group on order $n$.

If $\mathfrak{F}$ is a Fitting class, Lockett (1974) defines classes $\mathfrak{F}^{*}$ and $\mathfrak{F}_{*}$ by

$$
\begin{aligned}
& \mathfrak{F}^{*}=\left(G \in \subseteq: G \times G=(G \times G)_{\mathfrak{F}}(G \times 1)\right) ; \text { and } \\
& F_{*}=\cap\left(G: G \text { is a Fitting class with } \mathscr{G}^{*}=\mathfrak{F}^{*}\right),
\end{aligned}
$$

where $G \times G$ denotes the direct square of $G$. The following properties of the classes $\mathfrak{F}^{*}$ and $\mathfrak{F}_{*}$ are relevant here; these properties are proved in Lockett (1974), except where otherwise indicated.

2.1 THEOREM (Lockett). Let $\mathfrak{F}$ and (S) be Fitting classes. Then

(a) $\mathfrak{F}^{*}$ and $\mathfrak{F}_{*}$ are Fitting classes and

$$
\left(\mathfrak{F}_{*}\right)_{*}=\mathfrak{F}_{*} \subseteq \mathfrak{F} \subseteq \mathfrak{F}^{*}=\left(\mathfrak{F}^{*}\right)^{*}
$$

(b)

$$
\begin{aligned}
\mathfrak{F}_{*} \subseteq B_{S} \subseteq \mathfrak{F}^{*} & \text { if and only if } \mathscr{S}^{*}=\mathfrak{F}^{*}, \\
& \text { if and only if } \mathscr{G}_{*}=\mathfrak{F}_{*} ;
\end{aligned}
$$

(c) $\left(\mathfrak{F} \cap(\mathfrak{S})^{*}=\mathfrak{F}^{*} \cap \mathfrak{S}^{*}\right.$; 
(d) if $\mathfrak{F} \subseteq$ (S) then $\mathfrak{F}^{*} \subseteq \&^{*}$ and (Bryce and Cossey (1975), Corollary 3.5), $\mathfrak{F}_{*} \subseteq \mathfrak{G}_{*}$;

(e) if $\mathfrak{F}$ is closed under taking subgroups, quotient groups or subdirect products, then $\mathfrak{F}=\mathfrak{F}^{*}$;

(f) if $G$ and $H$ are groups then $(G \times H)_{\mathfrak{\Im}^{*}}=G_{\Im^{*}} \times H_{\Im^{*}}$;

(g) if $G \in \mathfrak{F}$ then $[G, \operatorname{Aut}(G)] \leqslant G_{\mathfrak{F}}$; and

(h) char $\left(\mathfrak{F}_{*}\right)=$ char( $(\mathfrak{F})$ and in particular, $\mathfrak{N} \subseteq \mathfrak{S}_{*}$.

A Fitting class $\mathfrak{F}$ with $\mathfrak{F}=\mathfrak{F}^{*}$ is called a Lockett class. A Fitting class $\mathfrak{F}$ with $\mathfrak{F} \supseteq \mathfrak{S}_{*}$ is called a normal Fitting class: it is shown in Lockett (1974), Theorem 2.2 , that this definition of normal Fitting class is equivalent to that given by Blessenohl and Gaschütz (1970), except that Belssenohl and Gaschütz allowed the trivial Fitting class, (1), to be considered normal.

If $\mathfrak{F}$ and $(\mathfrak{S}$ are Fitting classes, their product $\mathfrak{F} \# \mathbb{G}$ is defined to be

$$
\mathfrak{F} \#=\left(G \in \mathfrak{S}: G / G_{\mathfrak{F}} \in(\mathfrak{S})\right. \text {. }
$$

It is well-known that $\mathfrak{F} \#(S)$ is again a Fitting class, and that the product is associative. The following results of Cossey (1975) will be of use to us.

2.2 ThEOREM (Cossey). Let $\mathfrak{F}$ and \& be Fitting classes.

(a) If $\mathbb{G}$ is normal, then so also are $\mathfrak{F} \#(\mathbb{S}$ and $\mathbb{S} \# \mathfrak{F}$.

(b) If $G$ is a group with $G / G_{\mathfrak{F}} \notin \mathbb{S}_{*}$, then $G \notin \mathbb{S}_{*}$.

(c) If $\mathfrak{F}_{*}=\mathfrak{F}^{*} \cap \mathfrak{S}_{*}$, then $F \# \mathfrak{S}_{*}=\mathfrak{F}^{*} \# \mathfrak{S}_{*}$.

If $\pi$ is a set of primes and $\mathfrak{F}$ is a Fitting class, define

$$
\mathscr{K}_{\pi}(\mathfrak{F})=\left(G \in \subseteq: \operatorname{Hall}_{\pi}(G) \subseteq \mathfrak{F}\right) \text {. }
$$

It is easy to check that $K_{\pi}(\mathfrak{F})$ is again a Fitting class. Properties of $K_{\pi}(\mathfrak{F})$ are proved in Hauck (1978) and in Brison (1979); the next proposition lists some facts whose proofs may be found (where necessary) in the latter paper.

2.3 Proposition. Let $\mathfrak{F}$ and $\mathbb{S}$ be Fitting classes and let $\pi$ and $\sigma$ be sets of primes. Then

(a) $\mathscr{K}_{\pi}\left(\mathscr{K}_{\sigma}(\mathfrak{F})\right)=\mathscr{K}_{\pi \cap \sigma}(\mathfrak{F})$

(b) if $\mathfrak{F} \subseteq$ (S) then $\mathscr{K}_{\pi}(\mathfrak{F}) \subseteq \mathcal{K}_{\pi}(\mathbb{S})$;

(c) $\mathscr{K}_{\pi}\left(\mathfrak{F} \#(\mathbb{S})=\mathscr{K}_{\pi}(\mathfrak{F}) \# \mathscr{K}_{\pi}(\mathfrak{S})\right.$;

(d) $\mathscr{K}_{\pi}\left(\mathfrak{F}^{*}\right)=\left(\mathscr{K}_{\pi}(\mathfrak{F})\right)^{*}$;

(e) $\mathfrak{F}$ is Hall $\pi$-closed if and only if $\mathfrak{F} \subseteq \mathscr{K}_{\pi}(\mathfrak{F})$; and

(f) $\operatorname{char}\left(\mathscr{K}_{\pi}(\mathfrak{F})\right)=\pi^{\prime} \cup \operatorname{char}(\mathfrak{F})$.

It is shown in Brison (1979) how part (d) of this result may be used to deduce the following results of Bryce and Cossey (1975), Theorem 1.3 and Corollary 4.15. 
2.4 THEOREM (Bryce and Cossey). Let $\pi$ be a set of primes. Then

(a) the class $\widetilde{S}_{*}$ is Hall $\pi$-closed; and

(b) $\left(\mathfrak{S}_{\pi}\right)_{*}=\mathfrak{S}_{\pi} \cap \mathfrak{S}_{*}$.

We will use part (a) of this result without explicit mention.

The next fact will be crucial for the proofs of several of our results.

2.5 Theorem (Berger; Laue, Lausch and Pain, Bryant and Kovács; Cossey). Let $s$ and $t$ be distinct primes. Then $\mathfrak{S}_{s} \# \mathbb{S}_{t} \nsubseteq \mathfrak{S}_{*}$. Specifically, there exists $a$ group $H=H(s, t) \in \subseteq_{s} \# \Im_{t}$ such that $0_{t}(H)=1$, while $0_{s}(H)=H^{\prime}=H_{\Im}$, and $\left|H / 0_{s}(H)\right|=t$.

In the case where $t \mid s-1$, the existence of $H(s, t)$ in this theorem is shown by Berger (1976), Proposition 3. In the case where $t \nmid s-1$, the existence of $H(s, t)$ is a well-known consequence of the main theorems of the papers of Laue, Lausch and Pain (1977) and of Bryant and Kovács (1978); for example, a construction of a suitable group $H(s, t)$, given the results of Laue, Lausch and Pain and of Bryant and Kovács, is recorded in the thesis Brison (1978), Section 4.7.

If $G$ is any group and $n$ is a natural number, then $G^{n}$ will denote the (external) direct product of $n$ copies of $G$. Now suppose that $A$ and $B$ are groups, and let $W=B$ wr $A$ denote the regular wreath product of $B$ by $A$. Then we may regard $W$ as a split extension of a group $B^{*}$ by $A$, where $B^{*}=B^{|A|}$ is called the "base group" of $W$. If $C \leqslant B$, then $C^{*}$ will always denote the corresponding subgroup $C^{*}=\left\{\left(c_{1}, \ldots, c_{|A|}\right): c_{i} \in C\right\}$ of $B^{*}$. The following result is proved in Cossey (1975), Lemma 2.2 .

2.6 Lemma (Cossey). Let $\mathfrak{F}$ be a Lockett class and let $A$ and $B$ be groups. If $B \notin \mathfrak{F}$, then $(B \text { wr } A)_{\mathfrak{F}}=\left(B^{*}\right)_{\mathfrak{F}}=\left(B_{\mathfrak{F}}\right)^{*}$.

Finally we mention a well-known method for constructing groups with a unique chief series.

2.7 LEMMA. Let $G$ be a group with a unique minimal normal subgroup $N$, where $N \in \mathfrak{S}_{p}$ for some prime $p$. Let $q$ be a prime different from $p$. Then $G$ possesses a faithful irreducible $G F(q)$-module.

The above result follows because the regular $G F(q)$-module for $G$ is completely reducible as an $N$-module, by Maschke's Theorem. Repeated application of this lemma yields the following result. 
2.8 Corollary. Let $p_{1}, \ldots, p_{r}$ be a sequence of primes with $p_{i} \neq p_{i-1}$ for all relevant $i$. Then there exists a group $M$ with a unique chief series

$$
1=M_{r} \triangleleft M_{r-1} \triangleleft \cdots \triangleleft M_{1} \triangleleft M_{0}=M,
$$

and where $M_{i-1} / M_{i}$ is an elementary abelian $p_{i}$-group, $i=1, \ldots, r$. If $\left|M_{i-1} / M_{i}\right|=p_{i}^{a}$, then $a_{l}=1$, and we will denote such a group $M$ by $M=$ $M\left(p_{r}^{a_{3}}, \ldots, p_{1}\right)$.

\section{Preliminary results}

3.1 LeMMA. Let AT denote a semi-direct product of a group $A$ by a group $T$, where $|T|=t \in \mathbf{P}$, and suppose that $A=(A T)^{\prime}$. Let $D=\{(d, \ldots, d): d \in T\}$ $\leqslant(A T)^{t}$. Then $A^{t} D \in \Im_{*}$.

Proof. Let $C=\langle c\rangle$ denote a cyclic group of order $t$, and form $W=$ $(A T)$ wr $C$. We adhere to the notation for wreath products in Section 2. If $1 \leqslant i \leqslant t$ and $x \in A T$, then $x_{i}$ will denote the element

$$
x_{i}=\left(1, \ldots, x_{i}, \ldots, 1\right) \in(A T)^{*} .
$$

We may suppose notation chosen so that

$$
\begin{aligned}
x_{i}^{c}=x_{i+1}, \quad & 1 \leqslant i \leqslant t-1 ; \quad \text { and } \\
x_{t}^{c} & =x_{1},
\end{aligned}
$$

for all $x \in A T$.

Choose $d \in T$ with $d \neq 1$ and let $f=d_{1} \cdots d_{t} \in T^{*}$. Then $A^{*}\langle f\rangle \approx A^{\prime} D$ $\leqslant(A T)^{t}$. Since $d_{t}^{t}=1$, we have

$$
f=d_{1} d_{2}^{-1}\left(d_{2} d_{3}^{-1}\right)^{2} \cdots\left(d_{t-1} d_{t}^{-1}\right)^{t-1} .
$$

But $d_{i} d_{i+1}^{-1}=d_{i}\left(d_{i}^{c}\right)^{-1} \in W^{\prime}$ for all relevant $i$, and so $f \in W^{\prime}$. Since $A=(A T)^{\prime}$, then $A^{*}\langle f\rangle \leqslant W^{\prime} \in \subseteq_{*}$. But $W / A^{*} \cong C_{t}$ wr $C_{\mathrm{t}} \in \Im_{t}$, and so $A^{*}\langle f\rangle$ is subnormal in $W$. Thus $A^{*}\langle f\rangle \in \mathbb{S}_{*}$, and the result follows.

3.2 Lemma. Let $D$ be a group with subgroups $X, Y$ and $Z$ such that $D=X Y Z$ and $X \cap Y=1$. Suppose that $X \unlhd D$, that $Y \unlhd Y Z$ and that $C_{Y Z}(Y)<Y$. Let $\mathfrak{F}$ be a Fitting class with $(X Y)_{\mathfrak{F}}=X_{\mathfrak{F}}$. Then $D_{\mathfrak{F}}=X_{\mathfrak{F}}$.

Proof. Since $D_{\mathfrak{F}}$ and $X Y$ are normal in $D$, we have

$$
\left[D_{\overparen{F}}, X Y\right] \leqslant(X Y)_{\mathfrak{F}}=X_{\mathfrak{F}} \leqslant X,
$$


and so $D_{\mathfrak{F}} \leqslant J=C_{D}(X Y / X)$. But $X \leqslant J$, and so by Dedekind's law we have

$$
J=J \cap X Y Z=X\left(C_{Y Z}(X Y / X)\right)=X C_{Y Z}(Y),
$$

since $Y \unlhd Y Z$ and $X \cap Y=1$. But $C_{Y Z}(Y) \leqslant Y$, and so $D_{\mathscr{F}} \leqslant X Y$. Thus $D_{\mathfrak{F}}=(X Y)_{\mathfrak{F}}=X_{\mathfrak{F}}$, as claimed.

The next result follows from Satz 4.1 of Hauck (1979a).

3.3 THEOREM (Hauck). Let $\mathfrak{X}$ be a Fitting class and $\mathfrak{Y}$ be a Lockett class such that $\mathfrak{X} \subseteq \mathfrak{V}$. Let $p$ be a prime with $\mathfrak{X}^{*} \# \mathfrak{S}_{p} \nsubseteq \mathfrak{V}$. Then there exist groups $N \in \mathfrak{X}$ and $A \leqslant \operatorname{Aut}(N)$, with $|A|=p$, such that the semi-direct product $N A$ satisfies $N A \notin \mathfrak{V}$.

\section{Some products which are Hall-closed}

In this section we produce several examples of products of Fitting classes which are Hall $\pi$-closed. Our main interest is in products of the form $\mathfrak{F} \# \subseteq_{*}$; however, the first result disposes of products of the form $\mathfrak{S}_{*} \# \mathfrak{F}$, among others, and gives one direction of Theorem $D$.

4.1 Proposition. Let $\pi$ be a set of primes and let $\mathfrak{X}$ be a Hall $\pi$-closed Fitting class. If $\mathfrak{S}_{\pi} \subseteq \mathfrak{X}^{*}$, then $\mathfrak{X} \mathbb{F}$ is Hall $\pi$-closed for all Fitting classes $\mathfrak{F}$.

Proof. Let $G \in \mathfrak{X} \# \mathfrak{F}$, and let $H \in \operatorname{Hall}_{\pi}(G)$. Since $\mathfrak{X}$ is Hall $\pi$-closed then $H \cap G_{\mathfrak{x}} \leqslant H_{\mathfrak{x}}$. Now $H \in \mathfrak{X}^{*}$ and so $H_{\mathfrak{x}} \geqslant H^{\prime}$ by $2.1(\mathrm{f})$. But $H /\left(H \cap G_{\mathfrak{x}}\right) \cong$

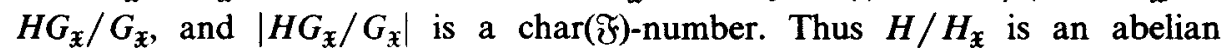
char( $\mathfrak{F})$-group, and so $H \in \mathfrak{X} \# \mathfrak{F}$. The result follows.

The hypotheses on $\mathfrak{X}$ in the above result are satisfied by any Hall $\pi$-closed normal Fitting class, and in particularly by the class $\mathfrak{S}_{*}$.

4.2 Lemma. Let $\mathfrak{D}$ be a Fitting class of characteristic $\sigma$, and let $\pi$ be a set of primes such that $\widetilde{S}_{\pi \cap 0} \subseteq \mathcal{D}^{*}$. Then

$$
\left(\mathfrak{D}^{*} \# \mathfrak{S}_{*}\right) \cap \mathfrak{S}_{\pi}=\left(\mathfrak{D} \# \Im_{*}\right) \cap \Im_{\pi} .
$$

Proof. Let $\delta=\pi \cap \sigma$. Now $\mathfrak{D}^{*} \cap \mathfrak{S}_{\pi}=\mathfrak{S}_{\delta}$ by the hypotheses, and so

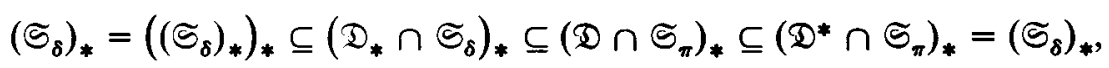


by 2.1(a) plus repeated application of 2.1(d). Thus

$\left(\mathfrak{D} \cap \mathfrak{S}_{\pi}\right)_{*}=\left(\mathfrak{S}_{\delta}\right)_{*}=\mathfrak{S}_{\delta} \cap \mathfrak{S}_{*}=\left(\mathfrak{D}^{*} \cap \mathfrak{S}_{\pi}\right) \cap \mathfrak{S}_{*}=\left(\mathfrak{D} \cap \mathfrak{S}_{\pi}\right)^{*} \cap \mathfrak{S}_{*}$,

the second equality by $2.4(\mathrm{~b})$ and the final equality by $2.1(\mathrm{c}, \mathrm{e})$. However,

$$
\begin{aligned}
& \left(\mathfrak{D}^{*} \# \mathfrak{S}_{*}\right) \cap \mathfrak{S}_{\pi}=\left(\left(\mathfrak{D} \cap \mathfrak{S}_{\pi}\right)^{*} \# \mathfrak{S}_{*}\right) \cap \mathfrak{S}_{\pi} ; \quad \text { and }
\end{aligned}
$$

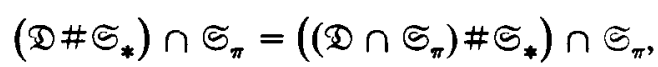

and so the assertion follows by Cossey's lemma 2.2(c).

The next result will, in Section 5, give one direction of Theorem A.

4.3 Proposition. Let $\mathfrak{D}$ be a Fitting class of characteristic $\sigma$, and let $\pi$ be a set of primes such that $\mathfrak{S}_{\pi \cap \sigma} \subseteq \mathfrak{D}^{*}$. Then $\mathfrak{D} \mathfrak{S}_{*}$ is Hall $\pi$-closed.

Proof. Let $\delta$ denote $\pi \cap \sigma$ and let $\mathfrak{I}$ denote $\mathfrak{S}_{\delta}$; then $\mathscr{D}^{*} \cap \mathfrak{S}_{\pi}=\mathfrak{T}$. Let $G \in \mathscr{D} \# \subseteq_{*}$, and let $H \in \operatorname{Hall}_{\pi}(G)$.

Set $K=H \cap G_{\Phi}$. Then $H / K \cong H G_{\Phi} / G_{\Phi} \in \Im_{*}$, since $\Im_{*}$ is Hall $\pi$-closed and $G / G_{\triangleright} \in \Im_{*}$. But now by $2.2(\mathrm{~b}),(H / K) /(H / K)_{\mathfrak{T}} \in \mathfrak{S}_{*}$. Since $K \in \mathfrak{T}$ and $(H / K)_{\mathbb{T}}=H_{\mathbb{T}} / K$, it follows that $H / H_{\mathbb{\Upsilon}} \in \mathfrak{S}_{*}$. Since $H_{\mathbb{\Upsilon}}=H_{\mathfrak{D}^{*}}$, it follows that $H \in \mathfrak{D}^{*} \# \subseteq_{*}$. Applying 4.2, we find that $H \in \mathfrak{D} \# \subseteq_{*}$, and the result follows.

The next result will be used in the final section to show how the conclusion of Theorem A (see Section 5) can break down if a hypothesis on the sets of primes involved is omitted.

4.4 Proposition. Suppose that the Fitting class $\mathfrak{X}$ and the sets $\pi$ and $\tau$ of primes satisfy either of the following conditions:

(a) $\tau \subseteq \pi$; or

(b) $\Im_{\tau \backslash\{p\}} \subseteq \mathfrak{X} \subseteq \Im_{\tau}$ for all $p \in \tau$.

Then if $\mathbb{B}$ is any Hall $\pi$-closed Fitting class, it follows that the class $\mathcal{K}_{\tau}(\mathfrak{X}) \# \mathbb{G}$ is also Hall $\pi$-closed.

Proof. By 2.3 we have

$$
\mathscr{K}_{\pi}\left(\mathscr{K}_{\tau}(\mathfrak{X}) \#(\mathbb{S})=\mathscr{K}_{\pi \cap \tau}(\mathfrak{X}) \# \mathscr{K}_{\pi}(\mathbb{S}) .\right.
$$

Suppose firstly that $\tau \subseteq \pi$. Since \& is Hall $\pi$-closed and $\tau=\pi \cap \tau$, it follows from (1) and 2.3(e) that

$$
\mathscr{K}_{\tau}(\mathfrak{X}) \# \mathbb{S} \subseteq \mathscr{K}_{\pi \cap \tau}(\mathfrak{X}) \# \mathscr{K}_{\pi}(\mathfrak{S})=\mathscr{K}_{\pi}\left(\mathscr{K}_{\tau}(\mathfrak{X}) \# \mathbb{S}\right),
$$

and so $\mathscr{K}_{\tau}(\mathfrak{X}) \#(\mathbb{S}$ is Hall $\pi$-closed. 
Suppose next that $\tau \nsubseteq \pi$, and that condition (b) holds. Then $\pi \cap \tau \subseteq \tau \backslash\{p\}$ for some $p \in \tau$, and so $\subseteq_{\pi \cap \tau} \subseteq \mathfrak{X}$. Thus $\subseteq \mathscr{K}_{\pi \cap \tau}\left(\mathscr{S}_{\pi \cap \tau}\right) \subseteq \mathscr{K}_{\pi \cap \tau}(\mathfrak{X})$, by 2.3 . But now by (1), we have

$$
\left(\mathscr{K}_{\tau}(\mathfrak{X}) \#(\mathfrak{S}) \subseteq \Im=\mathscr{K}_{\pi}\left(\mathcal{K}_{\tau}(\mathfrak{X}) \#(\mathfrak{S}),\right.\right.
$$

and the result follows.

This section on products which are Hall $\pi$-closed ends with the following result, which is very easy to check.

4.5 Proposition. Let $\pi$ be a set of primes and let $\mathfrak{F}$ and $\mathbb{E S}$ be Hall $\pi$-closed Fitting classes. If $\mathbb{B S} \cap \mathfrak{S}_{\pi}$ is closed under taking quotients, then $\mathfrak{F} \# \mathbb{G}$ is Hall $\pi$-closed.

\section{The proof of Theorem $A$}

Theorem A will be proved by a sequence of lemmas and constructions. Numbering of equations and other statements in the text will run consecutively through the section.

TheOREM A. Let $\mathfrak{D}$ be a Fitting class of characteristic $\sigma$, and let $\pi$ be a set of primes with $\pi \cup \sigma \neq \mathbf{P}$. Then $\mathfrak{D} \# \mathfrak{S}_{*}$ is Hall $\pi$-closed if and only if $\mathfrak{S}_{\pi \cap 0} \subseteq \mathfrak{D}^{*}$.

In Section 8, it is shown that the assertion of Theorem A can break down in the case that $\pi \cup \sigma=\mathbf{P}$.

We obtain the following immediate corollary.

5.1 Corollary. Let $\mathfrak{D}$ be a Fitting class of characteristic $\sigma$, where $\sigma \neq \mathbf{P}$. Then $\mathfrak{D} \# \widetilde{S}_{*}$ is Hall-closed if and only if $\mathfrak{D}^{*}=\widetilde{S}_{\sigma}$.

If $\subseteq_{\pi \cap \sigma} \subseteq \mathscr{D}^{*}$, where $\sigma=\operatorname{char}(\mathfrak{D})$, then it follows from 4.3 that $\mathfrak{D} \# \mathfrak{S}_{*}$ is Hall $\pi$-closed, and so it remains to prove that "only if" assertion of Theorem A.

We now fix for the rest of this section a Fitting class $\mathscr{D}$ of characteristic $\sigma$, and a set $\pi$ of primes with $\pi \cup \sigma \neq \mathbf{P}$ and $\mathfrak{S}_{\pi{ }_{0}} \nsubseteq \mathfrak{D}^{*}$. We shall construct a group which belongs to $\mathscr{D} \# S_{*}$, but which has a Hall $\pi$-subgroup which does not belong to $\mathfrak{D} \# \overleftarrow{S}_{*}$, and Theorem $A$ will follow.

Let $\mathfrak{F}=\mathfrak{D}^{*}$ and let $\delta=\pi \cap \sigma$. Since $\mathfrak{S}_{\delta} \nsubseteq \mathfrak{F}$, then $|\delta|>2$. 
5.2 LeMma. There exist groups $\bar{P}$ and $\bar{N}$, and groups $A_{1}<\operatorname{Aut}(\bar{P})$ and $A_{2}<$ $\operatorname{Aut}(\bar{N})$ with $\left|A_{1}\right|=\left|A_{2}\right|=t \in \delta$, such that

(i) $\bar{P}$ is an elementary abelian p-group, where $p \in \mathbf{P} \backslash(\pi \cap \sigma)$, with

$$
\bar{P}=\left[\bar{P}, A_{1}\right] \text { and }\left(\bar{P} A_{1}\right)_{\mathfrak{F}}=\left(\bar{P} A_{1}\right)_{\mathscr{D}}=1 ;
$$

and

(ii) $\left(\bar{N} A_{2}\right)_{\mathfrak{F}}=\left(\bar{N} A_{2}\right)_{\mathscr{D}}=\bar{N} \in \widetilde{S}_{\delta} \cap \mathfrak{D}$.

Here, of course, $\bar{P} A_{1}$ and $\bar{N} A_{2}$ denote the natural semi-direct products.

Proof. Since $\mathfrak{S}_{\delta} \nsubseteq \mathfrak{F}$, then there exists $t \in \delta$ with $\left(\mathfrak{F} \cap \mathfrak{S}_{\delta}\right) \# \mathfrak{S}_{t} \nsubseteq\left(\mathfrak{F} \cap \mathfrak{S}_{\delta}\right)$. Choose $p \in \mathbf{P} \backslash(\pi \cup \sigma)$, and let $\bar{P}$ be a faithful irreducible $G F(p)$-module for a cyclic group $A_{1}$ of order $t$ : such a module exists by 2.7 . We identify $A_{1}$ with the corresponding subgroup of $\operatorname{Aut}(\bar{P})$, and form $\bar{P} A_{1}$. Then $\bar{P}=\left[\bar{P}, A_{1}\right]$ and $\left(\bar{P} A_{1}\right)_{\mathfrak{F}}=\left(\bar{P} A_{2}\right)_{\mathscr{D}}={ }_{1}$.

By $2.1\left(\mathrm{c}\right.$, e), we have $\left(\mathfrak{D} \cap \mathfrak{S}_{\delta}\right)^{*}=\mathfrak{F} \cap \mathfrak{S}_{\delta}$, and so by 3.3 there exist groups $\bar{N} \in \mathfrak{D} \cap \mathfrak{S}_{\delta}$ and $A_{2} \leqslant \operatorname{Aut}(\bar{N})$ such that $\left|A_{2}\right|=t$ and $\bar{N} A_{2} \notin \mathfrak{F}$. But then $\left(\bar{N} A_{2}\right)_{\mathfrak{F}}=\left(\bar{N} A_{2}\right)_{\mathbb{D}}=N$. This completes the proof.

5.3 Construction. We take groups $\bar{P}, \vec{N}, A_{1}$ and $A_{2}$ as in the conclusion of 5.2.

Choose $s \in \delta$ with $s \neq t$ : this is possible because $|\delta| \geqslant 2$. By 2.5 there exists a group $S T \in \widetilde{S}_{s} \# \Im_{t} \backslash \widetilde{S}_{*}$ where $S=0_{s}(S T)=(S T)^{\prime}$ and $T \cong C_{t}$, while $0_{t}(S T)$ $=1$.

Let $Q$ denote either $\bar{P}$ or $\bar{N}$, and let $A$ denote either $A_{1}$ or $A_{2}$ according as $Q$ denotes $\bar{P}$ or $\bar{N}$ respectively. Form

$$
W=W(Q)=(Q A) \text { wr } S T,
$$

and let

$$
L=L(Q)=\left[A^{*}, S\right] \unlhd A^{*} S T,
$$

where $(Q A)^{*}=Q^{*} A^{*}$ denotes the base group of $W$ as in Section 2. Then $L=[L, S]$ by Gagen (1976), Corollary 0.2 , and so

$$
L S=(L S T)^{\prime} ; \text { and }
$$

$$
L=F(L S T), \text { the Fitting subgroup. }
$$

Now $(Q A)_{\mathfrak{F}}=Q_{\mathfrak{F}} \in \mathfrak{D}$, by 5.2 . Since $\mathfrak{F}$ is a Lockett class, then $(Q A)_{\mathfrak{F}}^{*}=$ $\left(Q_{\mathfrak{\mho}}\right)^{*}=Q_{\mathfrak{F}}^{*} \in \mathfrak{D}$. But $Q^{*} L \unlhd(Q A)^{*}$, and so $\left(Q^{*} L\right)_{\mathfrak{F}}=Q_{\mathfrak{\xi}}^{*}$.

Let $C=C(Q)=\left[Q^{*}, L S T\right]$. Then

$$
C=[C, L S T] \text {, }
$$

by Gagen (1976), Corollary 0.2. Now $C L \unlhd Q^{*} L$, and so

$$
(C L)_{F}=C L \cap\left(Q^{*} L\right)_{F}=C L \cap Q_{F}^{*}=C_{F} \in D,
$$


the final equality because $C \unlhd Q^{*}$ and $Q^{*} \cap L=1$. Since $C_{L S T}(L) \leqslant L$ by (2), then we may apply 3.2 with $X=C, Y=L$ and $Z=S T$ to conclude that $(C L S T)_{\mathfrak{F}}=C_{\Re}$.

Now set $P=C(\bar{P}) \leqslant \bar{P}^{*}$ and $N=C(\bar{N}) \leqslant \bar{N}^{*}$. Taking $Q$ successively equal to $\bar{P}$ and $\bar{N}$, we obtain groups

$$
G(\bar{P})=P L S T, \text { and } G(\bar{N})=N L S T,
$$

respectively, noting that $L(\bar{P})$ and $L(\bar{N})$ are isomorphic as $S T$-operator groups and can be identified, so that $L S T$ may be regarded as a group of operators on both $P$ and $N$. We note that

$$
(N L S T)_{\S}=N \in \mathfrak{D} .
$$

5.4 LEMMA. In the group PLST constructed above, we have $C_{L S T}(P)=1$.

Proof. In the notation of 5.3, and letting $A=A_{1}$, we have $1 \neq \bar{P}=[\bar{P}, A]$ and $|A|=t \in \mathbf{P}$. Thus $C_{A}(\bar{P})=1$, and so $C_{A^{*}}\left(\bar{P}^{*}\right)=1$. Suppose that au $\in$ $C_{A^{*} S T}\left(\bar{P}^{*}\right)$, where $a \in A^{*}$ and $u \in S T$, and suppose further that $u \neq 1$. Then by the wreath product construction, the element $u$ must move some component in the direct product $\bar{P}^{*}$. Since $A^{*}$ normalises each component of $\bar{P}^{*}$, then au must move some component, contrary to choice. Thus $u=1$ and so $C_{A^{*} S T}\left(\bar{P}^{*}\right)=$ $C_{A^{*}}\left(\bar{P}^{*}\right)=1$. Thus $C_{L S T}\left(\bar{P}^{*}\right)=1$. But $\bar{P}^{*}=C_{\bar{P}^{*}}(L S T) \times P$, by Gagen (1976), Corollary 0.5 , and so $C_{L S T}(P)=1$, as claimed.

5.5 Construction. We start with the group PLST of 5.3. Form (PLST), where $t=|T| \in \delta$. If $X \leqslant P L S T$ and $1 \leqslant i \leqslant t$, define the following subgroups of $(P L S T)^{t}$ :

$$
\begin{aligned}
X_{i} & =\{(1, \ldots, \ldots, \ldots, 1): x \in X\} ; \\
\hat{X}_{1} & =X_{2} \times \cdots \times X_{t} ; \\
X^{*} & =X_{1} \times \cdots \times X_{t} ; \text { and } \\
D & =\{(d, \ldots, d): d \in T\} .
\end{aligned}
$$

Note that $D$ has order $t$ and normalises each of the groups $P_{i}, L_{i}$ and $S_{i}$, and that $P_{i} L_{i} S_{i} D \cong P L S T$ for all relevant $i$.

Let $\Phi=(P L S)^{*} D$. It follows from (1) and (3) that $(P L S T)^{\prime}=P L S$. Thus by 3.1 we have

$$
\Phi \in \mathfrak{S}_{*} .
$$

Now let $K=P^{*} \hat{L}_{1} \hat{S}_{1} \unlhd \Phi$. Then $\Phi / K \cong L S T$. Now recall the group $N L S T$ constructed in 5.3. We may define an action of $\Phi / K$ on $N$ by requiring that $N(\Phi / K) \cong N L S T$. This action may be inflated to an action of $\Phi$ on $N$; let $\Theta$ denote the semidirect product $N \Phi$ so obtained. 
5.6 Proposition. The group $\Theta$ constructed above lies in both $\mathfrak{\#} \mathfrak{S}_{*}$ and $\mathfrak{D \#} \mathfrak{S}_{*}$, while a Hall $\pi$-subgroup of $\Theta$ lies in abelia of these classes.

Proof. We retain notation from 5.3-5.5.

Since $P_{\mathfrak{F}}=1$ and $N \in \mathfrak{F}$ then $\left(N \times P^{*}\right)_{\mathfrak{F}}=N$. Now, $C_{L S T}(P)=1$, by 5.4 , and so $C_{L^{*} S^{*} D}\left(P^{*}\right)=1$. Taking $X=N, Y=P^{*}$ and $Z=L^{*} S^{*} D$ in 3.2, we find that $\Theta_{\mathfrak{F}}=N$. Thus $\Theta / \Theta_{\mathfrak{F}} \cong \Phi \in \mathfrak{S}_{*}$. Since $N \in \mathfrak{D}$, we have

$$
\Theta \in \mathfrak{F} \# \mathfrak{S}_{*} \text { and } \Theta \in \mathfrak{D} \# \Im_{*} \text {. }
$$

Since $N \unlhd \Theta$ then $H=N L^{*} S^{*} D \leqslant \Theta$. By construction, $P \in \widetilde{S}_{\pi^{\prime}}$ and $H \in$ $\Im_{\pi} ;$ thus $H \in \operatorname{Hall}_{\pi}(\Theta)$. We may write $H=\left(N \hat{L}_{1} \hat{S}_{1}\right) L_{1} S_{1} D$. Now,

$$
\left(N \hat{L}_{1} \hat{S}_{1} L_{1}\right)_{\Re}=\left(N L_{1} \times \hat{L}_{1} \hat{S}_{1}\right)_{\mathfrak{F}}=N \times\left(\hat{L}_{1} \hat{S}_{1}\right)_{\Im}
$$

since $\mathfrak{F}$ is a Lockett class and $\left(N L_{1}\right)_{\mathfrak{F}}=N$ by (4). Further, $C_{L_{1} S_{1} D}\left(L_{1}\right)<L_{1}$ because of (2). Now taking $X=N \hat{L}_{1} \hat{S}_{1}, Y=L_{1}$ and $Z=S_{1} D$ in 3.2, we conclude that

$$
H_{\mathfrak{F}}=N \times\left(\hat{L}_{1} \hat{S}_{1}\right)_{\Im}
$$

Let $\mathbb{S}$ denote either $\mathfrak{D}$ or $\mathfrak{F}$. Since $\hat{L}_{1} \in \widetilde{S}_{\mathfrak{t}} \subseteq \mathfrak{D} \subseteq \mathfrak{F}$, then $N \times \hat{L}_{1}<H_{\mathscr{G}}<$ $N \times L_{1} S_{1}$. Thus $H / H_{\mathscr{G}}$ is isomorphic to a factor of $\left(L_{1} S_{1} \times \hat{S}_{1}\right) D$ by a normal subgroup, $M$ say, contained in $\hat{S}_{1}$. But then $\left(\left(L_{1} S_{1} \times \hat{S}_{1}\right) D / M\right)_{\mathfrak{R}}=$ $\left(L_{1} \times \hat{S}_{1}\right) / M$, and so

$$
\left(H / H_{\oplus S}\right) /\left(\left(H / H_{\Theta}\right)_{\Re}\right) \cong S_{1} D \cong S T .
$$

Since $S T \notin \Im_{*}$ by choice, it follows from $2.2(\mathrm{~b})$, that $H / H_{\mathscr{S}} \notin \Im_{*}$, and thus that

$$
H \notin \mathfrak{F} \# \mathfrak{S}_{*} \text { and } H \notin \mathfrak{D} \# \mathfrak{S}_{*} .
$$

This completes the proof of 5.6, and, with it, the proof of Theorem A.

\section{The proofs of Theorems B and G}

If $G \in \subseteq$ and $\pi \subseteq \mathbf{P}$, then $\pi$-soc $(G)$ denotes the product of all minimal normal $\pi$-subgroups of $G$, while $\operatorname{soc}(G)$ denotes P-soc $(G)$. Note that $\pi$-soc $(G)$ is a direct product of elementary abelian groups, and that $\operatorname{soc}(G)>1$ if $G>1$.

The proofs of Theorems $\mathrm{B}$ and $\mathrm{C}$ use the following construction.

6.1 Construction. The ingredients for the construction are as follows.

(a) Fitting classes $\mathfrak{F}$ and $\mathbb{B}$.

(b) A class $\mathscr{Q}$ of groups which is closed under taking quotients, and a Fitting class $\mathfrak{X}$ with $\mathscr{Q} \nsubseteq \mathfrak{X}$. 
(c) A group $T$ of minimal order in $\mathfrak{D} \backslash \mathfrak{X}$; clearly $T>1$.

(d) A set $\pi$ of primes with $T \in \mathbb{S}_{\pi}$.

(e) A group $G \in \mathfrak{F}$ with a normal subgroup $D \unlhd G$, such that $G / D \cong \operatorname{soc}(T)$, and with a subgroup $L \in \operatorname{Hall}_{\pi}(G)$ such that $L_{\mathscr{G}}=L_{\overparen{G}}=L \cap D$.

We shall construct a group $\Theta \in \mathfrak{F} \# \mathfrak{X}$, with a subgroup $\Phi \in \mathrm{Hall}_{\pi}(\Theta)$ such that $\Phi \notin B$ S $\#$.

To commence the construction, let $N$ denote $\operatorname{soc}(T)$. Then $G / D \cong N$ is a non-trivial abelian group. Let $\bar{T}$ denote $T / N$, and form the regular wreath product $W=G$ wr $\bar{T}$. In the usual notation for wreath products, $G^{*}=G^{|\bar{T}|}$ is the base group of $W$.

Now $W / D^{*} \cong(G / D)$ wr $\bar{T} \cong N$ wr $\bar{T}$, by elementary properties of the wreath product. By Huppert (1967), Kapitel I, Satz 15.9, there exists an injection $j: T \rightarrow W / D^{*}=\left(G^{*} \bar{T}\right) / D^{*}$ such that (by a reading of the proof),

$$
N j \leqslant G^{*} / D^{*} \text {. }
$$

Let $\Theta$ denote the complete pre-image in $W$ of $T j$; then $D^{*} \unlhd \Theta$ and $\Theta / D^{*} \cong$ $T$.

By construction, $L^{*}$ is a $\bar{T}$-invariant Hall $\pi$-subgroup of $G^{*}$, and so $L^{*} \bar{T} \in$ $\operatorname{Hall}_{\pi}(W)$.

Let $\Phi=\Theta \cap L^{*} \bar{T}$. Since $W / D^{*} \in \Im_{\pi}$, then the Hall $\pi$-subgroups of $W$ are conjugate under $D^{*} \leqslant \Theta$, and it follows that

$$
\Phi \in \operatorname{Hall}_{\pi}(\Theta) \text {, }
$$

and then that

$$
\Phi \cap G^{*}=\Phi \cap L^{*} .
$$

Let $K^{*}$ denote $D^{*} \cap L^{*}$, and note that $K^{*} \leqslant \Phi$. Then by (2),

$$
K^{*}=\Phi \cap D^{*} \cap L^{*}=\Phi \cap G^{*} \cap D^{*}=\Phi \cap D^{*} .
$$

Now $\Theta=D^{*} \Phi$ by considerations of orders, and so

$$
\Phi / K^{*} \cong \Theta / D^{*} \cong T \notin \mathfrak{X}
$$

But $\operatorname{soc}\left(\Theta / D^{*}\right) \leqslant\left(\Theta \cap G^{*}\right) / D^{*}$ by $(1)$, and so

$$
\operatorname{soc}\left(\Phi / K^{*}\right) \leqslant\left(\Phi \cap L^{*}\right) / K^{*},
$$

by (2) and the first isomorphism in (4).

By assumption, and the fact that $\mathscr{S}^{*}$ is a Lockett class, $K^{*}=L_{\mathscr{G}^{*}}^{*}$, and so $K^{*} \leqslant \Phi_{\mathfrak{G}^{*}}$. Suppose that $K^{*}<\Phi_{\mathfrak{G}^{*}}$. Then $\Phi_{\mathfrak{G} *} / K^{*} \cap \operatorname{soc}\left(\Phi / K^{*}\right) \# 1$. Since $L^{*} / K^{*}$ is abelian, it follows that $K^{*}<\Phi_{\mathscr{G}^{*}} \cap L^{*} \unlhd L^{*}$. However, $\Phi_{\mathbb{G}^{*}} \cap L^{*} \unlhd$ $\Phi_{\mathscr{G}}$ by (2), and so $\Phi_{\mathscr{G} *} \cap L^{*} \leqslant L_{\mathscr{G}^{*}}$, contrary to $K^{*}=L_{\mathfrak{G}^{*}}^{*}$ Thus $\Phi_{\mathfrak{G}^{*}}=K^{*} \in$ $\mathbb{B}$, and it follows that $\Phi \notin \mathbb{B} \#$.

Now, $G^{*} \cap \Theta \unlhd\left\langle G^{*}, \Theta\right\rangle(=W)$ because $G^{*} \unlhd W$, while $D^{*}\left\langle G^{*} \cap \Theta\right.$ and $G^{*} / D^{*}$ is abelian. Since $G^{*} \in \mathfrak{F}$, then $G^{*} \cap \Theta<\Theta_{\mathfrak{F}}$. But $\left(G^{*} \cap \Theta\right) / D^{*}>1$ by (1), and so $D^{*}<\Theta_{\mathfrak{H}}$. Since $\Theta / D^{*} \cong T$, it follows by the minimal choice of 
$T \in \mathfrak{Q} \backslash \mathfrak{X}$, and the fact that $\mathfrak{Q}$ is closed under taking quotients, that $\Theta / \Theta_{\mathfrak{Y}} \in \mathfrak{X}$. Thus $\Theta \in \mathfrak{F} \# \mathfrak{X}$.

Starting with the "ingredients" outlined above, we have now constructed a group $\Theta \in \mathfrak{F} \# \mathfrak{X}$, with a Hall $\pi$-subgroup $\Phi \notin(\mathfrak{G} \# \mathfrak{X}$. This completes the construction.

The above construction will now be used to prove two results about the Hall $\pi$-closure of products of Fitting classes, plus a "cancellation result."

Recall that if $\pi$ is a set of primes then the class

$$
3_{\pi}=(G \in \subseteq: \pi \text {-soc }(G) \leqslant Z(G))
$$

is a subdirect-product closed Fitting class, and so is a Lockett class in view of 2.1(e): see Bryce and Cossey (1974).

THEOREM B. Let $\mathfrak{X}$ be a Fitting class and $\pi$ be a set of primes with $|\pi| \geqslant 2$ and $\pi \neq \mathbf{P}$. Then $\mathfrak{F} \# \mathfrak{X}$ is Hall $\pi$-closed for all Fitting Classes $\mathfrak{F}$ if and only if $\mathfrak{\Im}_{\pi} \subseteq \mathfrak{X}$.

Proof. If $\mathfrak{S}_{\pi} \subseteq \mathfrak{X}$, then $\mathfrak{F} \# \mathfrak{X}$ is trivially Hall $\pi$-closed for all Fitting classes $\mathfrak{F}$, and so it remains to prove the converse.

Thus suppose that $\mathfrak{S}_{\pi} \nsubseteq \mathfrak{X}$, and let $T$ be a group of minimal order in $\mathfrak{S}_{\pi} \backslash \mathfrak{X}$. Let $N=\operatorname{soc}(T)$; then $N$ is non-trivial and is a direct product of its elementary abelian Sylow subgroups. Suppose that

$$
|N|=p_{1}^{n_{1}} \cdots p_{r}^{n_{2}}
$$

where the $p_{i}$ are distinct primes in $\pi$. For each $i \in\{1, \ldots, r\}$, choose $q_{i} \in \pi$ with $q_{i} \neq p_{i}$ : this is possible because $|\pi| \geqslant 2$. Choose $u \in \mathbf{P} \backslash \pi$. For each $i$, construct a group

$$
M_{i}=M\left(u^{b_{i}}, q_{i}^{a_{i}}, p_{i}\right),
$$

with unique chief series, as in 2.8. Since $u \notin \pi$ and $\operatorname{soc}\left(M_{i}\right)$ is a $u$-group, then $M_{i} \in Z_{\pi}$. If $H_{i} \in$ Hall $\left(M_{i}\right)$, then $\operatorname{soc}\left(H_{i}\right)$ is a non-central $q_{i}$-group, and so $H_{i} \notin 8_{\pi}$. Further, $M_{i} / M_{i}^{\prime} \cong C_{p_{i}}$.

Let $G=M_{1}^{n_{1}} \times \cdots \times M_{r}^{n_{4}}$, and let $D=G^{\prime}$. Then $G / D \cong N$. If $L \in$ Hall $_{\pi}(G)$, then $L \notin 8_{\pi}$, while the abelian group $L \cap D$ belongs to $3_{\pi}$.

Now perform construction 6.1 with $\mathfrak{F}=\mathbb{E}=3_{\pi}$, with $\mathfrak{Q}=\mathfrak{S}_{\pi}$, and with $\mathfrak{X}$, $T, \pi, G, D$ and $L$ as above. We obtain a group $\Theta \in \mathcal{Z}_{\pi} \# \mathfrak{X}$, having a Hall $\pi$-subgroup $\Phi \notin 3_{\pi} \# \mathfrak{X}$. Thus the class $3_{\pi} \# \mathfrak{X}$ is not Hall $\pi$-closed, and the theorem is proved.

We refer to Theorem $\mathrm{D}$ for comparison with Theorem $\mathrm{B}$.

It follows from the above that if $\mathfrak{F} \# \mathfrak{X}$ is Hall-closed for all Fitting classes $\mathfrak{F}$ (and fixed $\mathfrak{X}$ ), then $\mathfrak{X}=\mathfrak{S}$. 
As a second application of Construction 6.1, it will be shown that in certain circumstances, the Hall $\pi$-closure of a Lockett class $\mathfrak{F}$ can be inferred from that of $\mathfrak{F} \mathbb{X}$. We will need the following lemma, whose proof may be found in Bryce and Cossey (1974), Lemma 1.1.

6.2 LEMMA. Let $G$ be a group with normal subgroups $N, M$ such that $G / N M$ is nilpotent and $N \cap M=1$. Let $\mathfrak{F}$ be a Fitting class with $G / N \in \mathfrak{F}$. Then $G \in \mathfrak{F}$ if and only if $G / M \in \mathfrak{F}$.

6.3 Proposition. Let $\mathfrak{X}$ be a Fitting class of characteristic $\tau$, and let $\pi$ be a set of primes. Suppose that if $t \in \pi \cap \tau$, then there exists $s \in \pi \cap \tau$ with $\mathbb{S}_{t} \# \mathbb{S}_{s} \nsubseteq$ $\mathfrak{X}$. Let $\mathfrak{F}$ be a Lockett class such that $\mathfrak{F} \# \mathfrak{X}$ is Hall $\pi$-closed. Then $\mathfrak{F}$ is Hall $\pi$-closed.

Proof. Let $\left(\mathfrak{S}=\mathscr{K}_{\pi}(\mathfrak{F})\right.$; then $(\mathfrak{S})$ is a Lockett class by $2.3(\mathrm{~d})$. Suppose that $\mathfrak{F}$ is not Hall $\pi$-closed, and let $H$ be a group of minimal order in F\\&. Then $H$ has a unique maximal normal subgroup $E=H_{\mathscr{G}}$, which has index $t$ in $H$, for some $t \in \pi$. Let $M \in \operatorname{Hall}_{\pi}(G)$. Then $M \cap E=M_{\overparen{F}}=M_{\mathfrak{S}}$. There are two cases to consider.

Case 1. Suppose that $t \notin \tau$. Then let $T$ be a cyclic group of order $t$.

Case 2. Suppose that $t \in \tau$. Choose $s \in \pi \cap \tau$ with $\mathfrak{S}_{t} \# \subseteq_{s} \nsubseteq \mathfrak{X}$, and let $T$ be a group of minimal order in $\left(\mathfrak{S}_{t} \# \mathfrak{S}_{s}\right) \backslash \mathfrak{X}$. Then $T$ has a unique maximal normal subgroup, which coincides with $T_{\mathfrak{X}}$, and $\left|T: T_{\mathfrak{x}}\right|=s$. It follows that $T^{\prime} \in$ $\operatorname{Syl}_{t}(T)$, and then by 6.2 and the minimal choice if $T$, we find that $0_{s}(T)=1$.

In either case, $\operatorname{soc}(T)$ is elementary abelian of order $t^{n}$ for some $n>1$. Define $G=H^{n}$ and $D=E^{n}$. Define $L=M^{n}$; then $L \cap E=L_{\mathscr{S}}$ because $\mathscr{B}$ is a Lockett class. Let $\mathfrak{Q}=\widetilde{S}_{t}$ if Case 1 applies, and let $\mathfrak{Q}=S_{t} \# S_{s}$ if Case 2 applies. Now perform construction 6.1 with $\mathfrak{F}, \mathfrak{S}, \mathfrak{Q}, \mathfrak{X}, T, \pi, G, D$ and $L$ as defined here. We obtain a group $\Theta \in \mathfrak{F} \# \mathfrak{X}$ which has a Hall $\pi$-subgroup $\Phi \notin \mathbb{G} \# \mathfrak{X}$. Since $\mathfrak{S}_{\pi} \cap \mathfrak{F}=\mathfrak{S}_{\pi} \cap \mathfrak{G}$, by the definition of $\mathfrak{G}=\mathscr{K}_{\pi}(\mathfrak{F})$, it follows that $\Phi \notin \mathfrak{F} \# \mathfrak{X}$, and so $\mathfrak{F} \# \mathfrak{X}$ is not Hall $\pi$-closed. This contradiction completes the proof.

Note that by 2.5 , we may take $\mathfrak{X}=\mathfrak{S}_{*}$ in the above result.

We now come to the promised cancellation property for certain products of Fitting classes. We need a result of Hauck (1979b), Lemma 3.5, which for these purposes may be stated as follows.

6.4 Lemma (Hauck). Let $\mathfrak{F}$ and (S) be Fitting classes with $\mathfrak{F} \nsubseteq$ (S). Then there exists $H \in \mathfrak{F}$ such that $H_{\mathfrak{G}}=H_{\mathfrak{G} *}$ is the unique maximal normal subgroup of $H$. 
6.5 Proposition. Let $\mathfrak{F}$, $\mathscr{S}$ and $\mathfrak{X}$ be Fitting classes with $\mathfrak{F} \# \mathfrak{X} \subseteq \mathbb{B} \#$. Suppose that if $t \in \operatorname{char}(\mathfrak{X})$ then there exists $s \in \operatorname{char}(\mathfrak{X})$ with $\mathfrak{S}_{t} \# \mathfrak{S}_{s} \nsubseteq \mathfrak{X}$. Then $\mathfrak{F}^{*} \subseteq \mathbb{S S}^{*}$.

Proof. Suppose that $\mathfrak{F} \nsubseteq S^{*}$. Let $H \in \mathfrak{F}$ be as in the conclusion of 6.4; then $C=H_{\mathscr{G}}=H_{\mathfrak{G}^{*}}$ has prime index $t$ in $H$. If $t \notin \operatorname{char}(X)$ then $H \in$ $(\mathfrak{F} \# \mathfrak{X}) \backslash(\mathfrak{G} \# \mathfrak{X})$, contrary to assumption. Thus $t \in \operatorname{char}(\mathfrak{X})$, and so there exists $s \in \operatorname{char}(\mathfrak{X})$ with $\Im_{1} \# \Im_{s} \nsubseteq \mathfrak{X}$. Let $\mathfrak{Q}=\Im_{t} \# \Im_{s}$, and let $T$ be a group of minimal order in $\mathfrak{X} \mathfrak{X}$. As in the proof of $6.3, \operatorname{soc}(T)$ is elementary abelian of order $t^{n}$, where $n \geqslant 1$.

With $n$ as just defined, let $G=H^{n}$, and let $D=C^{n} \leqslant G$. Then $G / D \cong$ $\operatorname{soc}(T)$, while since $\mathscr{S}^{*}$ is a Lockett class then $G_{G^{*}}=D \in(G$.

Now perform construction 6.1 with $\mathfrak{F}, \mathbb{S}, \mathfrak{Q}, \mathfrak{X}, T, G$ and $D$ as above, and with $\pi=\mathbf{P}$, and $L=G$. We obtain a group $\Theta=\Phi \in \mathrm{Hall}_{\pi}(\Theta)$ such that $\Theta \in(\mathfrak{F} \# \mathfrak{X}) \backslash(\mathfrak{S} \# \mathfrak{X})$, contrary to hypothesis. Thus $\mathfrak{F} \subseteq\left(S^{*}\right.$, and so $\mathfrak{F}^{*} \subseteq \mathbb{S}^{*}$. This completes the proof.

It is not hard to find classes satisfying the hypothesis on $\mathfrak{X}$ in 6.5 ; for example, we may take $\mathfrak{X}=\mathfrak{N}$, the class of nilpotent groups, or, more generally, $\mathfrak{X}=\mathfrak{Y} \times$ 3 , where $\mathfrak{V}$ and 8 are non-trivial Fitting classes of co-prime characteristics. It may also be verified that the class of groups with central socle satisfies the hypothesis on $\mathfrak{X}$. Possibly the most interesting consequence of 6.5 is given in Theorem $\mathrm{C}$ below. Theorem $\mathrm{C}$ has been proved in the case that $\mathfrak{X}_{*}=\varsigma_{*}$ by Hauck (1979a), Satz 6.1, and Hauck's result had originally been conjectured by Cossey (1975), page 293.

THEOREM C. Let $\mathfrak{X}$ be a Fitting class with $|\operatorname{char}(\mathfrak{X})| \geqslant 2$. Let $\mathfrak{F}$ and (S) be Fitting

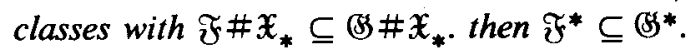

Proof. If $s$ and $t$ are distinct primes in $\operatorname{char}(\mathfrak{X})$, then $\mathfrak{S}_{t} \# \mathbb{S}_{s} \nsubseteq \mathfrak{S}_{*}$ by 2.5 , while $\mathfrak{X}_{*} \subseteq \Im_{*}$ by $2.1(\mathrm{~d})$. The result now follows immediately from 6.5 .

\section{The proof of Theorem D}

We start by recalling the definition of a certain family of Fitting classes. While these classes are "well-known", and are due to Gaschütz, they seem tohave been sparsely documented in the literature, except in the thesis of Lockett (1971). 
Thus let $\mathfrak{F}$ be a Fitting class and $q$ be a prime. Define

$$
e_{q}(\mathfrak{F})=\left(G \in \mathfrak{S} \text { : The } G \text {-chief } q \text {-factors of } G \text { lying in } G_{\mathfrak{F}} \text { are } G\right. \text {-central). }
$$

It is proved in Lockett (1971), Proposition 2.2.1, that $e_{q}(\mathfrak{F})$ is a Lockett class.

THEOREM D. Let $\mathfrak{X}$ be a Fitting class and $\pi$ he a set of primes with $|\pi| \geqslant 2$ and $\pi \neq \mathbf{P}$. Then $\mathfrak{X} \mathbb{F}$ is Hall $\pi$-closed for all Fitting classes $\mathfrak{F}$ if and only if $\mathfrak{X}$ is Hall $\pi$-closed and $\mathfrak{S}_{\pi} \subseteq \mathfrak{X}^{*}$.

Proof. The "if" assertion has been proved in 4.1.

Suppose that $\mathfrak{X} \mathbb{F}$ is Hall $\pi$-closed for all Fitting classes $\mathfrak{F}$. Then $\mathfrak{X}=\mathfrak{X} \#(1)$ is Hall $\pi$-closed, where (1) denotes the Fitting class of groups of order 1 .

Let $\mathfrak{U}=\mathfrak{X}^{*}$, and suppose for a contradiction that $\varsigma_{\pi} \nsubseteq \mathfrak{A}$. By 6.4, there exists a group $H \in \widetilde{S}_{\pi}$ such that $H_{\mathfrak{X}}=H_{\mathfrak{X}}$ is the unique maximal normal subgroup of $H$. Then $\left|H: H_{\mathfrak{Q}}\right|=p \in \pi$, and $H_{\mathfrak{Q}} \geqslant H^{\prime}$.

Choose $q \in \pi \backslash\{p\}$, and $r \in \mathbf{P} \backslash \pi$. Construct a group $M=M\left(r^{b}, q^{a}, p\right)$ as in 2.8 , with a unique chief series

$$
1<M_{2}<M_{1}<M, \text { for some } a, b \in \mathbf{N} .
$$

Form the regular wreath product $W=H$ wr $M$, with base group $W^{*}$ in the usual notation. By 2.6 , it follows that

$$
W_{\mathfrak{Y}}=\left(H^{*}\right)_{\mathfrak{A}}=\left(H_{\mathfrak{Y}}\right)^{*} \in \mathfrak{X},
$$

and so $W_{\mathfrak{X}}=W_{\mathfrak{X}}$ and $W / W_{\mathfrak{X}} \cong C_{p}$ wr $M$. Let $\mathfrak{R}^{2}$ denote $\mathfrak{R} \#$, the class of meta-nilpotent groups. It is easy to check that

$$
\left(C_{p} \text { wr } M\right)_{\mathfrak{R}^{2}}=\left(C_{p}\right)^{*} M_{2} \text {. }
$$

But now

$$
\left(W / W_{\mathfrak{X}}\right)_{\mathfrak{R}^{2}}=H^{*} M_{2} / W_{\mathfrak{X}} \in S_{q^{\prime}} .
$$

Thus $W / W_{\mathfrak{x}} \in e_{q}\left(\mathfrak{N}^{2}\right)$, and so $W \in \mathfrak{X} \# e_{q}\left(\mathfrak{R}^{2}\right)$.

Let $K \in \operatorname{Hall}_{\pi}(M)$. Then $L=H^{*} K \in \operatorname{Hall}_{\pi}(W)$. If $L_{\mathfrak{X}} \$ H^{*}$, then, as in the proof of 3.2 , we find that

$$
C_{M}\left(H^{*} / H_{\mathfrak{x}}^{*}\right) \geqslant C\left(H^{*} / H_{\mathfrak{x}}^{*}\right)>1,
$$

which is impossible by the wreath product construction. Thus $L_{\mathfrak{X}}=H_{\mathfrak{X}}^{*}$ and $\left(L / L_{\mathfrak{X}}\right)_{\mathfrak{R}^{2}}=\left(H^{*} Q\right) / L_{\mathfrak{X}}$, where $Q \in \operatorname{Syl}_{q}(K)$. But $Q \$ Z(K)$ by construction of $M$, and so $L / L_{\mathfrak{X}} \notin e_{q}\left(\mathfrak{R}^{2}\right)$. But now $L \notin \mathfrak{X} \# e_{q}\left(\mathfrak{R}^{2}\right)$, and so $\mathfrak{X} \# e_{q}\left(\mathfrak{R}^{2}\right)$ is not Hall $\pi$-closed, contrary to assumption. This completes the proof.

7.1 Corollary. Let $\mathfrak{X}$ be a Fitting class. Then $\mathfrak{X} \mathfrak{F}$ is Hall-closed for all Fitting classes $\mathfrak{F}$ if and only if $\mathfrak{X}$ is a Hall-closed normal Fitting class. 


\section{Concluding remarks}

The paper by Cusack (1980) contains several results on the Hall $\pi$-closure of normal Fitting classes. Theorem 1 of Cusack's paper states that if $\pi$ is any set of primes with $|\pi| \geqslant 2$ and $\pi \neq \mathbf{P}$, then there exists a normal fitting class which is not Hall $\pi$-closed. This result follows from each of our Theorems A and B, although the class produced by Cusack is different to any of ours. Theorem 5 of Cusack's paper gives a criterion for the Hall $\pi$-closure of any normal Fitting class in terms of the "join" operation, where if $\mathfrak{F}$ and $\mathbb{B S}$ are Fitting classes then their join $\mathfrak{F} \vee \&$ is the smallest Fitting class which contains both $\mathfrak{F}$ and $\mathbb{S}$. In fact, slight modifications to Cusack's proof, plus the use of certain properties of the $\mathscr{K}_{\pi}($ ) operator listed in Brison (1979), yield a criterion for the Hall $\pi$-closure of an arbitrary Fitting class, as follows.

8.1 TheORem (Cusack). Let $\mathfrak{F}$ be a Fitting class and $\pi$ be a set of primes. Then $\mathfrak{F}$ is Hall $\pi$-closed if and only if

$$
\mathfrak{F}=\left(\mathfrak{S}_{\pi} \cap \mathfrak{F}\right) \vee\left(\mathscr{K}_{\pi}\left(\mathfrak{F}_{*}\right) \cap \mathfrak{F}\right)
$$

This result may be put together with certain of our results, to perhaps shed some light on the join operation.

Finally, recall that Theorem A carries a restriction on the sets of primes involved, namely that $\pi \cup \sigma \neq \mathbf{P}$, in the notation of Section 5. It might be of interest to determine the full picture, and we mention here some fragments of progress in that direction. Because of 4.3 , the restriction is not needed for the "if" part of Theorem A, but the following examples show that some restriction is necessary for the "only if" part.

Firstly, let $p$ be a prime and $\mathscr{B}$ be a Fitting class. Note that $\mathscr{K}_{p}(1)=\Im_{p^{\prime}}$. Taking $\mathfrak{X}=(1)$ and $\tau=\{p\}$ in $4.4(\mathrm{~b})$, we find that $\overleftarrow{S}_{p^{\prime}} \#(S)$ is Hall $\pi$-closed whenever $\mathbb{G}$ is. In particular, it follows from 4.3 that $\left(\widetilde{S}_{p^{\prime}} \# \Im_{p}\right) \# \Im_{*}$ is Hall $\pi$-closed for all $\pi \subseteq \mathbf{P}$. In this case, $\sigma=\pi \cup \sigma=\mathbf{P}$, and certainly $\varsigma_{\pi \cap \sigma}=\varsigma_{\pi} \nsubseteq$ $\left(\Im_{p} \# \Im_{p}\right)^{*}$ whenever $\pi$ properly contains $\{p\}$.

Next let $\pi=\{p, q, r\}$ and $\rho=\{p, q\}$, where $p, q$ and $r$ are distinct primes. Taking $\pi=\tau$ in 4.4(a), we find that the class $\mathscr{K}_{\pi}\left(\mathfrak{R}_{\rho}\right) \# \Im_{*}$ is Hall $\pi$-closed. In this case, $\sigma=\operatorname{char}\left(\mathscr{K}_{\pi}\left(\mathfrak{R}_{\rho}\right)\right)=\pi^{\prime} \cup \rho$, and so $\pi \cup \sigma=\mathbf{P}$. However, $\pi \cap \sigma=\rho$, and it is clear that $\widetilde{S}_{\rho} \nsubseteq \mathscr{K}_{\pi}\left(\mathfrak{N}_{\rho}\right)=\left(\mathscr{K}_{\pi}\left(\mathfrak{R}_{\rho}\right)\right)^{*}$, the latter equality because of 2.1(e) and 2.3(d).

We note that if $\mathfrak{X}, \pi$ and $\tau$ satisfy $4.4(a)$ or (b), then either $\mathfrak{X}=(1), \tau=\{p\}$ and $\mathscr{K}_{\tau}(\mathfrak{X})=\subseteq_{p^{\prime}}$, or else $\pi \cup \operatorname{char}\left(\mathscr{K}_{\tau}(\mathfrak{X})\right)=\mathbf{P}$.

The above examples show how Proposition 4.4 may be used to manufacture Fitting classes $\mathfrak{D}$ of characteristic $\sigma$ such that $\mathscr{D} \# \subseteq_{*}$ is Hall $\pi$-closed, where 
$\pi \cup \sigma=\mathbf{P}$, and $\mathfrak{S}_{\pi \cap \sigma} \nsubseteq \mathfrak{D}^{*}$. Another approach is to try to find conditions on the fitting class $\mathfrak{D}$ which ensure that $\mathfrak{D} \# \mathfrak{S}_{*}$ is not Hall $\pi$-closed, and we now restrict attention to this aspect.

Some immediate information is provided by Proposition 6.3: if $\mathfrak{F}$ is a Lockett class which is not Hall $\pi$-closed, then $\mathfrak{F} \# S_{*}$ is not Hall $\pi$-closed. In the thesis Brison (1978), it is shown that $\mathfrak{D} \# \subseteq_{*}$ is not Hall-closed for a number of Fitting classes $\mathfrak{D}$ of full characteristic. One result in this direction is the following, which is proved by an elaboration of the proof of Theorem A above: see Brison (1978), Theorem 3.3.1.

8.2 Proposition. Let $\mathfrak{D}$ be a Fitting class of characteristic $\mathbf{P}$, and suppose that there exist groups $A, B \in S_{\mathfrak{D}}$ * with $(|A|,|B|)=1$. Then $\mathfrak{D} \# \mathbb{S}_{*}$ is not Hallclosed.

In this context, it is not hard to check that if $\mathfrak{X}, \pi$ and $\tau$ satisfy $4.4(\mathrm{~b})$, then $t \mid$ $|A|$ whenever $t \in \tau$ and $A \in \mathcal{S} \backslash \mathcal{K}_{\tau}(\mathfrak{X})$.

Entering the realm of special cases, there is a familiar class not covered by anything so far. If $p$ is a prime and $n$ is a natural number, let $\mathfrak{R}_{p}(n)$ denote the class of (finite, soluble) groups of $p$-length at most $n$. It is shown in Brison (1978), Proposition 3.4.2, that $\mathfrak{R}_{p}(n) \# \Im_{*}$ is not Hall-closed. By inserting the full strength of 2.5 above into the argument, it may in fact be shown that $\mathfrak{I}_{p}(n) \# \Im_{*}$ is not Hall $\pi$-closed whenever $p \in \pi,|\pi| \geqslant 2$ and $\pi \neq \mathbf{P}$.

\section{References}

J. C. Beidleman (1977), 'On products and normal Fitting classes', Arch. Math. (Basel) 28, 347-356.

Thomas R. Berger (1976), 'More normal Fitting classes of finite solvable groups", Math. Z. 151, 1-3. Dieter Blessenohl und Wolfgang Gaschütz (1970), 'Über normale Schunck- und Fittingklassen', Math. Z. 118, 1-8.

Owen John Brison (1978), On the theory of Fitting classes of finite groups (Ph.D. thesis, University of Warwick).

Owen J. Brison (1979), 'Hall operators for Fitting classes', Arch. Math. (Basel) 33, 1-9.

R. M. Bryant and L. G. Kovacs (1978), 'Lie representations and groups of prime power order', $J$. London Math. Soc. (2) 17, 415-421.

R. A. Bryce and John Cossey (1974), 'Metanilpotent Fitting classes', J. Austral. Math. Soc. 17, 285-304.

R. A. Bryce and John Cossey (1975), 'A problem in the theory of normal Fitting classes', Math. Z. 141, 99-110.

John Cossey (1975), 'Products of Fitting classes', Math. Z. 141, 289-295.

Elspeth Cusack (1980), 'Normal Fitting classes and Hall subgroups', Bull. Austral. Math. Soc. 21, 229-236. 
Terence M. Gagen (1976), Topics in finite gromps (London Mathematical Society Lecture Notes Series 16, Cambridge).

B. Hartley (1969), 'On Fischer's dualization of formation theory', Proc. London Math. Soc. (3) 19, 193-207.

Peter Hauck (1978), 'Eine Bemerkung zur kleinsten normalen Fittingklasse', J. Algebra 53, 395-401.

Peter Hauck (1979a), 'Fittingklassen und Kranzprodukte', J. Algebra 59, 313-329.

Peter Hauck (1979b), 'On products of Fitting classes', J. London Math. Soc. (2) 20, 423-434.

B. Huppert (1967), Endliche Gruppen I (Springer-Verlag, Berlin, Heidleberg, New York).

Hartmut Laue, Hans Lausch und Garry R. Pain (1977), 'Verlagerung und normale Fittingklassen endlicher Gruppen', Math. Z. 154, 257-260.

F. P. Lockett (1971), On the theory of Fitting classes of finite soluble groups (Ph.D. thesis, University of Warwick).

F. Peter Lockett (1974), 'The Fitting class $\mathfrak{F}^{* \prime}$, Math. Z. 137, 131-136.

Seç̧ão de Matemática Pura

Faculdade de Ciências

Avenida 24 de Julho, 134, $3^{\circ}$.

1.300 Lisboa

Portugal 